ARTICLE

https://doi.org/10.1038/s41467-019-09806-6

\title{
Genetics and evidence for balancing selection of a sex-linked colour polymorphism in a songbird
}

Kang-Wook Kim (1) 1,8, Benjamin C. Jackson (1) 1,8, Hanyuan Zhang1, David P.L. Toews (1) 2,3,4,

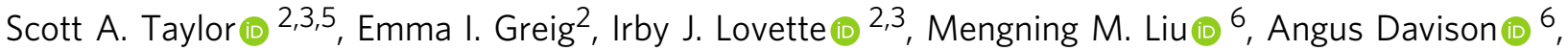
Simon C. Griffith (10 ${ }^{7}$, Kai Zeng (i) ${ }^{1} \&$ Terry Burke (i) ${ }^{1}$

Colour polymorphisms play a key role in sexual selection and speciation, yet the mechanisms that generate and maintain them are not fully understood. Here, we use genomic and transcriptomic tools to identify the precise genetic architecture and evolutionary history of a sexlinked colour polymorphism in the Gouldian finch Erythrura gouldiae that is also accompanied by remarkable differences in behaviour and physiology. We find that differences in colour are associated with an $\sim 72-\mathrm{kbp}$ region of the $\mathrm{Z}$ chromosome in a putative regulatory region for follistatin, an antagonist of the TGF- $\beta$ superfamily genes. The region is highly differentiated between morphs, unlike the rest of the genome, yet we find no evidence that an inversion is involved in maintaining the distinct haplotypes. Coalescent simulations confirm that there is elevated nucleotide diversity and an excess of intermediate frequency alleles at this locus. We conclude that this pleiotropic colour polymorphism is most probably maintained by balancing selection.

\footnotetext{
${ }^{1}$ Department of Animal and Plant Sciences, University of Sheffield, Sheffield S10 2TN, UK. ${ }^{2}$ Fuller Evolutionary Biology Program, Cornell Lab of Ornithology, Cornell University, 159 Sapsucker Woods Road, Ithaca, NY 14850, USA. ${ }^{3}$ Department of Ecology and Evolutionary Biology, Cornell University, Corson Hall, Ithaca, NY 14853, USA. ${ }^{4}$ Department of Biology, The Pennsylvania State University, University Park, PA 16802, USA. ${ }^{5}$ Department of Ecology and Evolutionary Biology, University of Colorado Boulder, Boulder, CO 80309, USA. ${ }^{6}$ School of Biology, University of Nottingham, University Park, NG7 2RD Nottingham, UK. ${ }^{7}$ Department of Biological Sciences, Macquarie University, Sydney, NSW 2109, Australia. ${ }^{8}$ These authors contributed equally: Kang-Wook Kim, Benjamin C. Jackson. Correspondence and requests for materials should be addressed to K.-W.K. (email: k.kim@sheffield.ac.uk) or to K.Z. (email: k.zeng@sheffield.ac.uk) or to T.B. (email: t.a.burke@sheffield.ac.uk)
} 
C olour-polymorphic species are observed across diverse taxa, but are relatively rare and younger than monomorphic species ${ }^{1,2}$. This is probably because any difference in fitness between alternative morphs should result in either the fixation of one of the causative alleles under natural or sexual selection, or lead to sympatric speciation under disruptive selection-potentially increasing the rate of speciation and promoting the transition from polymorphism to monomorphism ${ }^{2-4}$. Even if there are no fitness differences, genetic drift is expected to remove alternative morphs from a small population ${ }^{3}$. Therefore, the evolutionary processes that maintain colour polymorphisms in some species over long periods are not well understood. This is partly because the genetic basis of pigmentation, especially for non-melanic pigments such as carotenoids and psittacofulvins, has only recently been elucidated ${ }^{5-8}$.

Gouldian finch (Erythrura gouldiae) head plumage is a classic example of a Mendelian colour polymorphism in which the morphs co-occur in sympatry. The striking head colour morphs of the Gouldian finch reflect variation in both carotenoid and melanin pigmentation ${ }^{9-11}$; a pigmentation locus on the $\mathrm{Z}$ chromosome (hereafter the Red locus, with a recessive $Z^{r}$ allele and a less frequent dominant $Z^{R}$ allele) produces melanin-based black ( $Z^{r} Z^{r}$ in male, $Z^{r}$ in female) or carotenoid-based red $\left(Z^{R} Z^{R}, Z^{R} Z^{r}\right.$ in male, $Z^{R}$ in female) head morphs in both sexes, as well as differences in feather structure ${ }^{9,10}$. Rarely in the wild $(<1 \%$ of birds), an epistatic interaction of the Red locus with an autosomal locus produces a yellow morph ${ }^{11,12}$.

With regards to the black and red morphs, there are remarkable inter-morph differences in stress hormone levels, personalities, social dominance, sperm-length plasticity and sex allocation associated with the colour polymorphism (see Supplementary Table 1 for summary and references). Moreover, pre- and postzygotic incompatibilities have been documented between these morphs in a captive population (Supplementary Table 1), but the same phenomena have not been fully investigated in wild populations ${ }^{13}$.

Despite these differences, red and black morphs coexist in natural populations at stable phenotypic frequencies ${ }^{12}$, suggesting that there are mechanisms maintaining this multi-functional colour polymorphism in nature. Given that multiple phenotypes are associated with head colour, one hypothesis is that the Red locus is a supergene, defined as a tight cluster of genes acting as a mechanical unit in particular allelic combinations ${ }^{14}$, which is often facilitated by a chromosomal inversion, as recently demonstrated in several avian species ${ }^{15-18}$. Alternatively, the Red locus might be a single pleiotropic locus that can regulate multiple genes ${ }^{19}$. We undertake a detailed analysis of the region associated with the colour polymorphism using genomic and transcriptomic tools, and identify the genomic location of the Red locus as a putative regulatory region for follistatin on the $\mathrm{Z}$ chromosome. In addition, by using coalescent simulations, we find evidence that balancing selection is the most probable evolutionary mechanism behind the maintenance of the colour polymorphism in the Gouldian finch.

\section{Results and Discussion}

Identification of the Red locus. By using high-depth/low SNP density RADSeq ${ }^{20}$ and low-depth/high SNP density wholegenome sequencing (see Methods), we identified a region on the $\mathrm{Z}$ chromosome strongly associated with red-black head colouration (1000 permutations, $P<0.05$, Fig. 1a, Supplementary Figs. 1 and 2) within the candidate interval identified in a previous linkage analysis ${ }^{21}$. The genotypes of the wild birds $\left(n_{\text {male }}=\right.$ $89, n_{\text {female }}=72$ ) from an allele-specific PCR in this region showed a near-perfect association with head colour, with one exception, in a female (Fisher's exact test, male: $P<10^{-15}$; female: $P=$ $7.52 \times 10^{-10}$, Fig. 1b, Supplementary Fig. 2). The frequencies of the black-linked allele in this population were similar in females (0.88) and males (0.85) (Fisher's exact test, $P=0.69)$. The genotypic frequencies in males did not depart from Hardy-Weinberg Equilibrium $\left(P_{\text {permutation }}=0.24\right.$, Supplementary Table 3$)$. The low frequency of homozygous red males is not significantly different from expectation for a sex-linked locus under random mating (Supplementary Table 3) and a prediction based on the phenotypic frequency ${ }^{9}$.

High LD but no genomic rearrangements around the Red locus. Next, we examined if the multi-functional polymorphism is caused by a supergene. Previously-described supergenes have large genomic blocks of elevated linkage disequilibrium (LD) that harbour multiple genes. We measured the extent of LD around the Red locus, defined as the region with the most significant association with the colour polymorphism and its flanking regions (Fig. 1c), using Sanger sequencing of multiple loci. Intriguingly, the LD block significantly associated with the colour polymorphism was limited to a single intergenic region (where no known protein-coding genes are annotated in zebra finch) that spans $\sim 72$ kilobase pairs (kbp) and lies between the coding regions of the genes molybdenum cofactor synthesis 2 (MOCS2) and follistatin (FST) (Fig. 1c). In this region, the density of fixed differences between the $Z^{R}$ and $Z^{r}$ alleles was high (6.8-27.8 per kbp; Supplementary Table 5). This region, which is also supported by the analyses of diversity, divergence and simulations (below), is hereafter considered to comprise the Red locus.

Centromeres and genomic rearrangements, particularly chromosomal inversions, may reduce recombination rates and generate significant LD blocks between haplotypes ${ }^{22}$. However, our previous work showed that the Red locus is not located near the centromere or in a large genomic rearrangement between morphs ${ }^{21}$. We confirmed this finding with a fine-scale test for inversion breakpoints using long-range PCR: our genomic assembly across the Red locus for each genotype contained contiguous homologous sequence, consistent with the absence of an inversion differentiating the $Z^{R}$ and $Z^{r}$ alleles (Fig. 1c, Supplementary Table 7).

The Red locus as a candidate regulatory region of FST. Gouldian finch head colour is associated with multiple phenotypic traits (see above). As such, the intergenic location of the Red locus suggests that this locus acts as a regulatory region that controls a pleiotropic gene. From a small-scale transcriptomic analysis of regenerating feather follicles from the scalp, we identified a list of differentially expressed genes belonging to functionally correlated gene ontology (GO) networks with potential roles in feather development and pigmentation (Supplementary Fig. 3, Supplementary Data 1). Although neither MOCS2 nor FST were differentially expressed between morphs, some genes in this network are known to interact with FST (Supplementary Fig. 3, Supplementary Data 1).

The multiple differences in gene expression suggest that the fates of feather precursors are determined by gene(s) that act upstream of the regulatory network at an earlier developmental stage. Previous studies indicate that FST is a strong candidate for regulating the colour polymorphism. Follistatin is known to regulate hair and feather development through its antagonistic action on genes of the TGF- $\beta$ superfamily ${ }^{23,24}$. The genes in this family (e.g. activin, TGF- $\beta$ and BMP) may control melanocyte differentiation and melanogenesis ${ }^{25,26}$. FST and its downstream genes might also be responsible for the structural differences in the feathers found in the Gouldian finch polymorphism as $B M P$ and its alternative antagonist, noggin, are important 
a

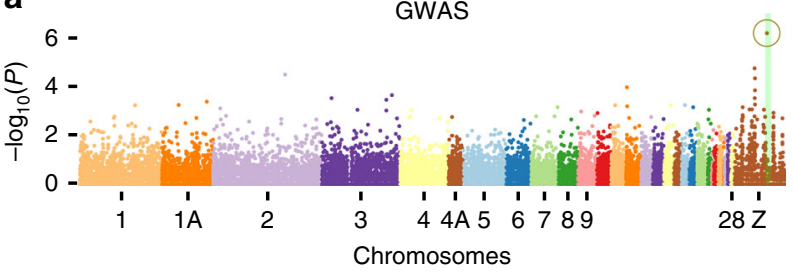

b

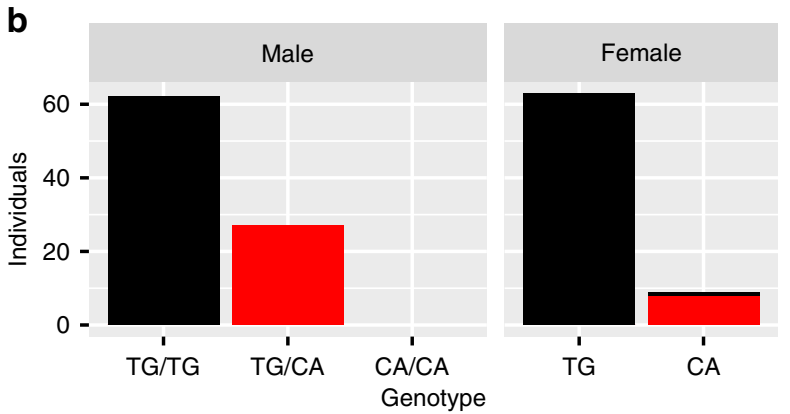

C
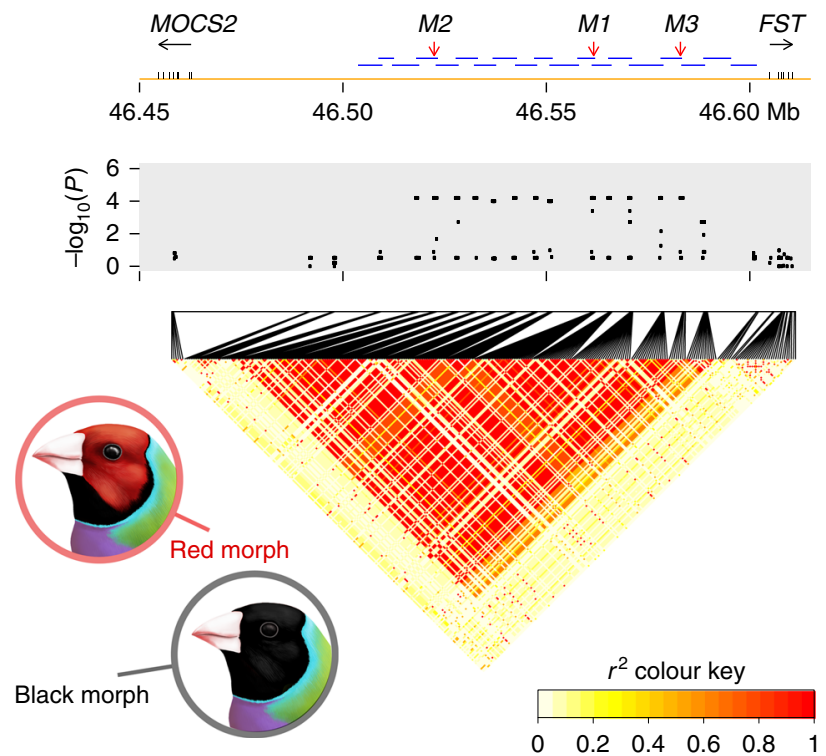

Fig. 1 Identification of the Red locus. a Association between RADSeq markers and head colour in Gouldian finches $\left(n_{\text {black }}=22, n_{\text {red }}=10\right)$. A circle around a dot indicates the only significant signal in GWAS $(P<0.05,1000$ permutations). The region shaded in green on the $Z$ chromosome represents the candidate region $(\sim 7.2 \mathrm{cM})$ based on a previous linkage mapping study 21 , where the Red locus co-segregated perfectly with microsatellite markers. b Genotypes of 161 birds from a wild population (male: $n_{\text {black }}=62, n_{\text {red }}=27$; female: $n_{\text {black }}=64, n_{\text {red }}=8$ ) based on two contiguous SNPs in the Red locus, where bar colours represent head colour. These SNPs (TG/CA) were identified by RADSeq (M1 at 46,561,597-46,561,598 bp in the zebra finch) and were typed using allele-specific PCR (Supplementary Fig. 2, Supplementary Table 2). c The extent of linkage disequilibrium (LD) associated with the colour trait. In the top panel, the locations of the protein-coding regions on the zebra finch Z chromosome (Mb) are indicated by vertical bars on the horizontal axis and the horizontal arrows below the gene names indicate the direction of transcription. Horizontal blue bars show the overlapping long-range PCR products (totalling $100 \mathrm{kbp}$ ) that were subsequently sequenced, which includes the 72-kbp region with elevated LD defined as the Red locus in this study (see Fig. 2, Supplementary Tables 6, 7). The three vertical red arrows indicate the locations of markers (M1-M3) that were used to genotype the wild birds in association tests (Supplementary Fig. 2, Supplementary Table 2). The middle panel shows $-\log _{10} P$ for association tests between phenotype and 282 SNPs from 26 fragments in 16 females ( $n_{\text {black }}=8, n_{\text {red }}=8$ ) typed using Sanger sequencing. The lower panel shows pairwise LD $\left(r^{2}\right)$ between SNPs. Finch illustrations by Megan Bishop

determinants of the morphogenesis of feather branching ${ }^{24,27}$. The TGF- $\beta$ superfamily and its regulator FST are also involved in the development and regulation of the visual ${ }^{28,29}$ and reproductive systems ${ }^{30}$.

We found no differences in the FST protein-coding sequences between black- and red-headed birds, and the amino-acid sequences were conserved between the Gouldian finch and the zebra finch (Supplementary Fig. 4). However, we identified several evolutionarily conserved sites within the Red locus located upstream of FST (Supplementary Fig. 5), suggesting that this region has functional importance. It is therefore likely that cisregulatory region(s) in the Red locus determine spatio-temporal variation in the expression of FST that then controls multiple genes in the TGF- $\beta$ superfamily, which in turn may be responsible for the pigmentation difference and other pleiotropic effects. We note that one of the few divergent regions that distinguish the genomes of hybridizing golden-winged (Vermivora chrysoptera) and blue-winged ( $V$. cyanoptera) warblers has been mapped to the same intergenic region between MOCS2 and FST ${ }^{31}$, suggesting convergent evolution of plumage colouration mechanisms and a possibly important role of the Red locus as a barrier to gene flow in other taxa. However, the fact that this locus has not led to speciation in the Gouldian finch indicates that selection may instead promote the persistence of divergent morphs in this species.

The nucleotide diversity and divergence at the Red locus. If selection has played a role in the origin and maintenance of the Red locus then it may have left a signature in the pattern of nucleotide diversity in this region. We calculated point estimates of summary statistics at the Red locus, as well as in slidingwindows across the $\sim 100-\mathrm{kbp}$ sequenced candidate region that included the Red locus to characterize the pattern of nucleotide diversity and divergence within and between morphs (Fig. 2a-d; Supplementary Table 9). We then compared the Red locus with 24 randomly-chosen intronic loci on the $\mathrm{Z}$ chromosome, as a putatively neutrally-evolving reference set (Fig. 2e-h; Supplementary Table 9). Notably, diversity for the combined sample of alleles ( $Z^{R}$ and $Z^{r}$ together) at the Red locus is elevated compared with the reference loci, as is the level of differentiation between $Z^{R}$ and $Z^{r}$ alleles.

The mean nucleotide diversity at the reference loci $\left(\pi_{\text {ref }}\right)$ was 0.0033 and varied little between the alleles of each morph (Fig. 2e). The point estimate of nucleotide diversity for the combined sample at the Red locus $\left(\pi_{\text {total }}=0.0102\right)$ was above that at all 24 reference loci (Fig. 2a), while diversity within the $Z^{R}$ $\left(\pi_{\text {red }}=0.0004\right)$ and the $Z^{r}\left(\pi_{\text {black }}=0.0015\right)$ alleles was similar to that at the reference loci.

The estimate of absolute divergence $d_{\mathrm{xy}}$ (the mean number of pairwise differences per site ${ }^{32}$ ) between the $Z^{R}$ and $Z^{r}$ alleles at the Red locus $\left(d_{\mathrm{xy} \text { Red locus }}=0.019\right)$ was again greater than between morphs at all 24 reference loci $\left(d_{\mathrm{xy} \mathrm{ref}}=0.0033\right.$; Fig. $\left.2 \mathrm{~b}, \mathrm{f}\right)$ and the estimate of net divergence $d_{\mathrm{a}}\left(d_{\mathrm{xy}}\right.$ minus the mean of the two within-morph $\pi$ values) was not greatly different from $d_{\mathrm{xy}}$ due to the low nucleotide diversity within each allelic class (data not shown). In contrast, the divergence between zebra finch and alleles of each morph was similar at both the Red locus and the reference loci (Fig. 2b, f). 
a $0.03-$

\& 0.015 -

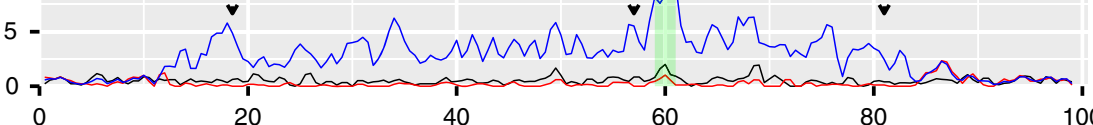

b $\quad 0.1$ -
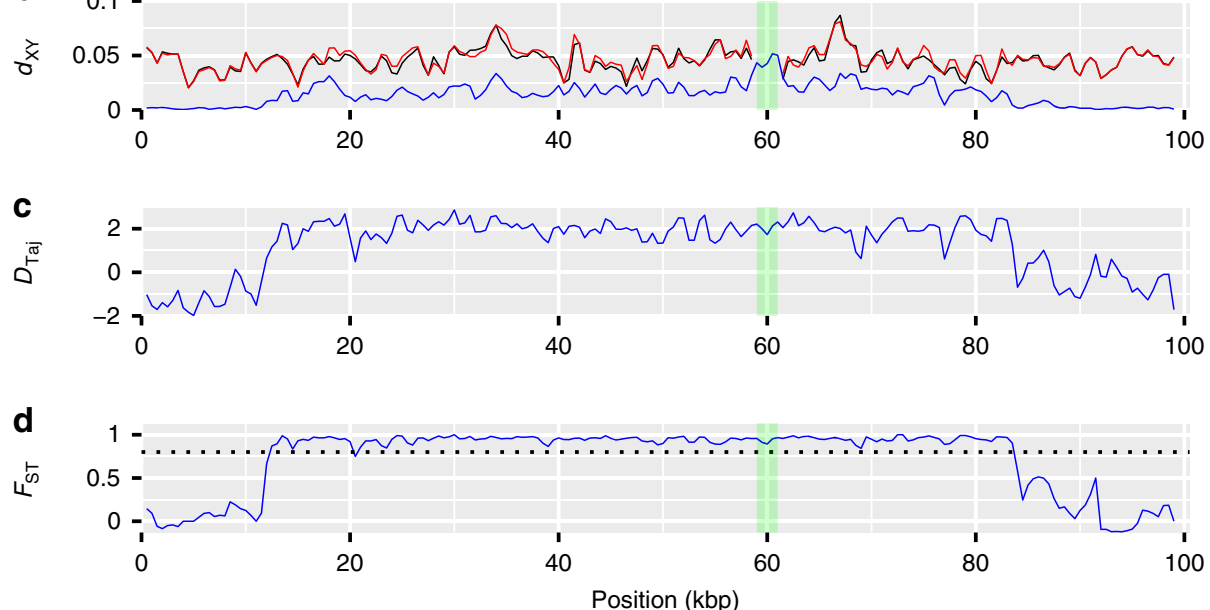

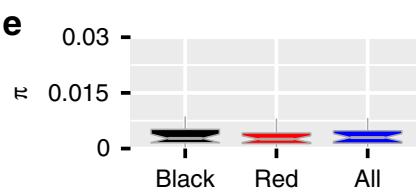

f
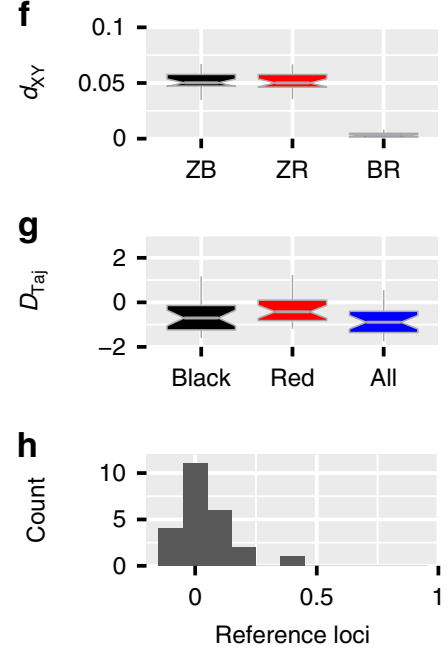

Fig. 2 Diversity and divergence at the Red locus and reference loci. Position in a-d represents $\sim 100 \mathrm{kbp}$ alignment of assembled MiSeq reads for 12 females ( $n_{\text {black }}=6, n_{\text {red }}=6$ ), which corresponds to the position between $46,503,706-46,601,744$ bp in the zebra finch reference genome (the region connected by horizontal blue bars in Fig. 1c). a-d Sliding-window analysis (window size of 1000 bp with a step of 500 bp) was used to obtain estimates of summary statistics. Green shading indicates an insertion of a putative LTR retrotransposon in the Gouldian finch that is not found in zebra finch. a Nucleotide diversity $(\pi)$ of black (black line), red (red line) morphs and all samples combined (blue line). b Sequence divergence between zebra finch and each morph (ZB: black line; ZR: red line), and between morphs (BR: blue line). c Tajima's $D$ for all samples combined. $\mathbf{d} F_{S T}$ between morphs. A horizontal dashed line indicates $F_{\mathrm{ST}}=0.8$. The data in $\mathbf{e}-\mathbf{h}$ were based on 24 intronic reference loci on the $Z$ chromosome, with $\mathbf{e}-\mathbf{g}$ showing the same summary statistics as $\mathbf{a}-\mathbf{c}$ and $\mathbf{h}$ a histogram of $F_{\mathrm{ST}}$. The box in $\mathbf{e}-\mathbf{g}$ bounds the interquartile range with a notch showing a $95 \%$ confidence interval around the median, and Tukey-style whiskers extend to a maximum of $1.5 \times$ interquartile range beyond the box. We define the 72 -kbp sequenced region with $F_{\mathrm{ST}}>0.8$ in $\mathbf{d}$ as the Red locus in subsequent analyses

Tajima's $D^{33}$ was moderately negative at the reference loci, both within the alleles of each morph and with both morphs' alleles combined $\left(D_{\text {Taj black }}=-0.599, D_{\text {Taj }}\right.$ red $=-0.304$ and $\left.D_{\text {Taj total }}=-0.818\right)$, as well as within the alleles of each morph at the Red locus $\left(D_{\text {Taj red }}=-0.844\right.$ and $D_{\text {Taj black }}=-0.891$; Fig. $2 \mathrm{~g}$, Supplementary Table 9), consistent with population expansion (see Supplementary Methods). However, $D_{\text {Taj }}$ was positive in the combined sample at the Red locus $\left(D_{\text {Taj total }}=2.193\right.$; Fig. $\left.2 \mathrm{c}\right)$, which is significantly $>0$ under the standard neutral model $\left(P_{\text {coalescent }}=0.002\right)$ using simulations in DnaSP v5 $5^{34}$, and again higher than at all 24 reference loci.

In captive populations of the Gouldian finch, pronounced preand postzygotic incompatibilities between morphs have been described, and mixed-morph pairings resulted in reduced survival: $40 \%$ of male and $80 \%$ of female offspring died before 90 days of age $\mathrm{e}^{35,36}$. If these incompatibilities persisted in the wild, then genome-wide differentiation between the black and red morphs might be expected. To quantify this, we estimated Wright's fixation index $F_{\mathrm{ST}}^{37}$ between each morph's alleles. $F_{\mathrm{ST}}$ between alleles of each morph at the Red locus reached a nearmaximal value $\left(F_{\mathrm{ST}}=0.951\right)$ and was significantly $>0\left(P_{\text {permutation }}\right.$ $=0.003)$. This value was greater than $F_{\mathrm{ST}}$ between each morph's alleles at all the Z-linked reference loci (composite $F_{\mathrm{ST}}=0.038$, Fig. 2d, h, Supplementary Fig. 1).

In contrast, we found no genomic differentiation with regards to colour morph across the genome outside of the Red locus in the wild sample. First, a coancestry matrix ${ }^{38}$ constructed for 32 wild birds $\left(n_{\text {black }}=22, n_{\text {red }}=10\right)$ using autosomal RADSeq loci, designed to fully exploit the available haplotypic information, failed to identify any clusters that separated the morphs
(Supplementary Fig. 6). Second, a STRUCTURE ${ }^{39}$ analysis between morphs using nine unlinked autosomal microsatellite markers for all the wild birds $\left(n_{\text {black }}=126, n_{\text {red }}=35\right)$ indicated that the most-likely number of subpopulations was one $(K=1$; Supplementary Fig. 7). These results suggest that there is no or little incompatibility leading to any genome-wide differentiation between morphs in the wild.

Test for neutrality. Overall, the patterns of nucleotide diversity and divergence at the Red locus (i.e. elevated $\pi$, excess of intermediate frequency alleles and maximal $F_{\mathrm{ST}}$ ) are consistent with balancing selection ${ }^{40}$. However, such patterns can be obtained even under neutral evolution if sampling is conditioned on a site associated with the phenotypes of interest, as in this case (e.g. see the simulated patterns of polymorphism close to the focal site presented in Fig. 3). Therefore, to formally test for neutrality we carried out coalescent simulations under a neutral model that incorporated demography, recombination, and accounted for our sampling scheme and asked if the pattern of genetic variation we observed within the 100-kbp-region is compatible with neutral expectations (see Methods and Supplementary Methods, Supplementary Figs. 8-17, Supplementary Tables 11-14 for detailed procedures of the neutrality test).

Our simulations show that the observed patterns of genetic variation at the Red locus cannot be explained by either neutral processes or biases caused by the sampling scheme (Fig. 3, Supplementary Methods). Assuming the $Z^{R}$ allele is derived (i.e., the derived allele frequency, DAF $=0.144$ ), the observed nucleotide diversity $\left(\pi_{\text {total }}\right)$ and Tajima's $D$ for the combined sample of 
a
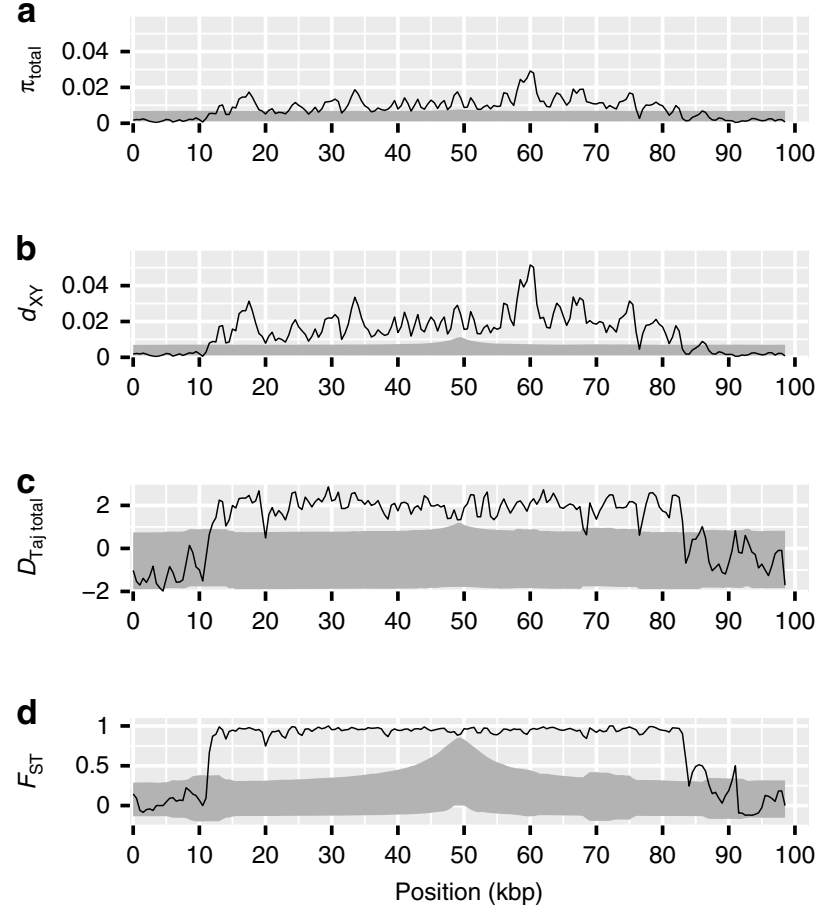

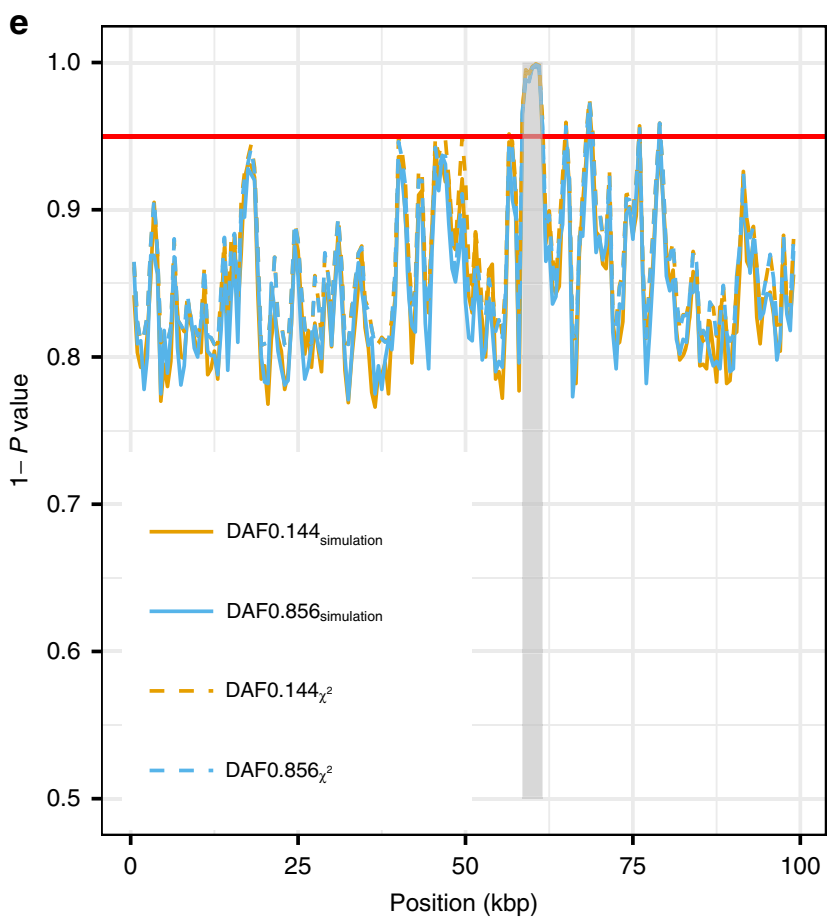

Fig. 3 Test for neutrality using coalescent simulations. Position in a-e represents $\sim 100 \mathrm{kbp}$ alignment of assembled MiSeq reads for 12 females $\left(n_{\text {black }}=6\right.$, $n_{\text {red }}=6$ ) as in Fig. 2. a-d Shown are sliding-window comparisons of the observed polymorphism at the Red locus with the polymorphism simulated under a neutral model that incorporated the sampling scheme (six alleles for each of the red and black morphs), demography, recombination, and a derived allele frequency (DAF) of 0.144 at the focal site (see Supplementary Methods). Solid lines represent the observed data (cf. Fig. 2) and the grey-shaded regions represent the space encompassed by the $95 \%$ confidence intervals derived from the simulations. a Nucleotide diversity for both morphs' alleles combined. b $d_{\mathrm{XY}}$ between the two morphs' alleles. c Tajima's $D$ for both morphs' alleles combined. $\mathbf{d} F_{\mathrm{ST}}$ between the two morphs' alleles across the Red locus. e A sliding-window implementation of the modified HKA test, taking into account the observed sampling scheme, allele frequencies, mutation rate and recombination rate. One minus the $P$-value is plotted for each window for DAF of 0.144 (yellow lines) and 0.856 (blue lines), using the chi-squared distribution (dashed lines) and simulations (solid lines). The red horizontal line indicates a $P$-value threshold of 0.05 . The shaded grey bar indicates an insertion of a putative LTR retrotransposon in the Gouldian finch that is not found in zebra finch

$Z^{R}$ and $Z^{r}$ alleles at the Red locus $\left(D_{\text {Taj total }}\right)$ both lie above the $95 \%$ confidence intervals (CIs) of the simulations for most windows, as do $F_{\mathrm{ST}}$ and $d_{\mathrm{xy}}$ between the $Z^{R}$ and $Z^{r}$ alleles (Fig. 3a-d). In contrast, statistics calculated within the $Z^{R}$ or $Z^{r}$ alleles fell within the $95 \%$ CIs of the simulated distributions, with the exception of nucleotide diversity within the $Z^{R}$ allele $\left(\pi_{\mathrm{red}}\right.$, Supplementary Fig. 9). The same pattern was observed when we assumed that the $Z^{r}$ allele was derived (DAF $=0.856$, Supplementary Fig. 10). In particular, the observed region of greater than expected differentiation extended over an $\sim 72-\mathrm{kbp}$ interval, with a rapid drop on either side-contrasting with neutral expectations for a single site on which sampling is conditioned, which results in a gradual decline towards background levels over a $20-\mathrm{kbp}$ interval (Fig. 3d). As we have demonstrated here, there is no evidence of an inversion across this region (Supplementary Table 7). Moreover, we found evidence for the presence of recombination within the Red locus (Supplementary Table 13, Supplementary Methods). Therefore, the highly diverged haplotype blocks are not likely to be maintained by reduced recombination, but rather by selection against recombinants. A sliding-window implementation of the HKA test ${ }^{41}$, taking into account nonrandom sampling, demography and recombination at the Red locus, also revealed significant departures from neutrality for multiple windows across the locus (Fig. 3e).

The evolutionary history of the black and red haplotypes. Balancing selection can maintain polymorphism over extended periods of time, resulting in deeply diverged haplotypes that may be even older than the species in which they reside ${ }^{40}$. In a gene genealogy of the M1 region (see Fig. 1c) for 14 species in the family Estrildidae, sequence divergence between black and red haplotypes was deeper than that between other species or even between genera (Fig. 4, Supplementary Fig. 18). Four other Erythrura species formed a monophyletic group (bootstrap support $100 \%)$, separate from the Gouldian finch, whereas none of the Gouldian finch haplotypes clustered with the sequences of red-faced finches (E. pealii and E. psittacea) or blue-faced finches (E. tricolor and E. trichroa). Despite the high divergence, the two Gouldian finch haplotypes formed a clear monophyletic group (bootstrap support 100\%), distinct from other Erythrura species, suggesting that these haplotypes evolved within the Gouldian finch lineage (Fig. 4). These patterns suggest that the divergent haplotypes at the Red locus are old (nearly half of the divergence between zebra finch and the Gouldian finch), although we did not attempt to estimate the age since balancing selection is likely to affect this estimation. Near the highly divergent $M 1$ region, we identified an insertion with the characteristic feature of a long terminal repeat (LTR) retrotransposon, which is absent in the zebra finch (Fig. 2). Transposable elements are often associated with adaptive polymorphism ${ }^{42}$. Indeed, signals of selection, including the departure of the observed data from expectations under neutrality derived from simulations as well as from the HKA test, were strongest in this region (Fig. 3e, Supplementary Methods). However, comparison of the $3^{\prime}$ and $5^{\prime}$ LTRs in each haplotype suggests that the divergence between the colour-associated haplotypes did not occur until after the 


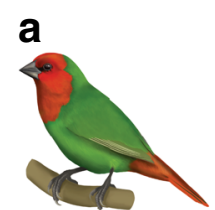

E. psittacea

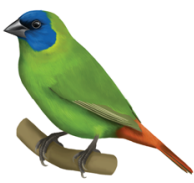

E. trichroa

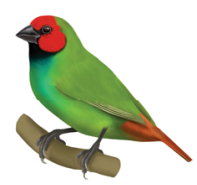

E. pealii

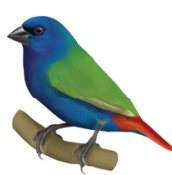

E. tricolor

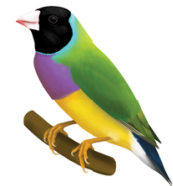

E. gouldiae (black)

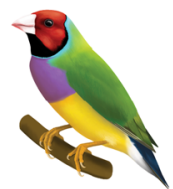

E. gouldiae (red)

\section{b}

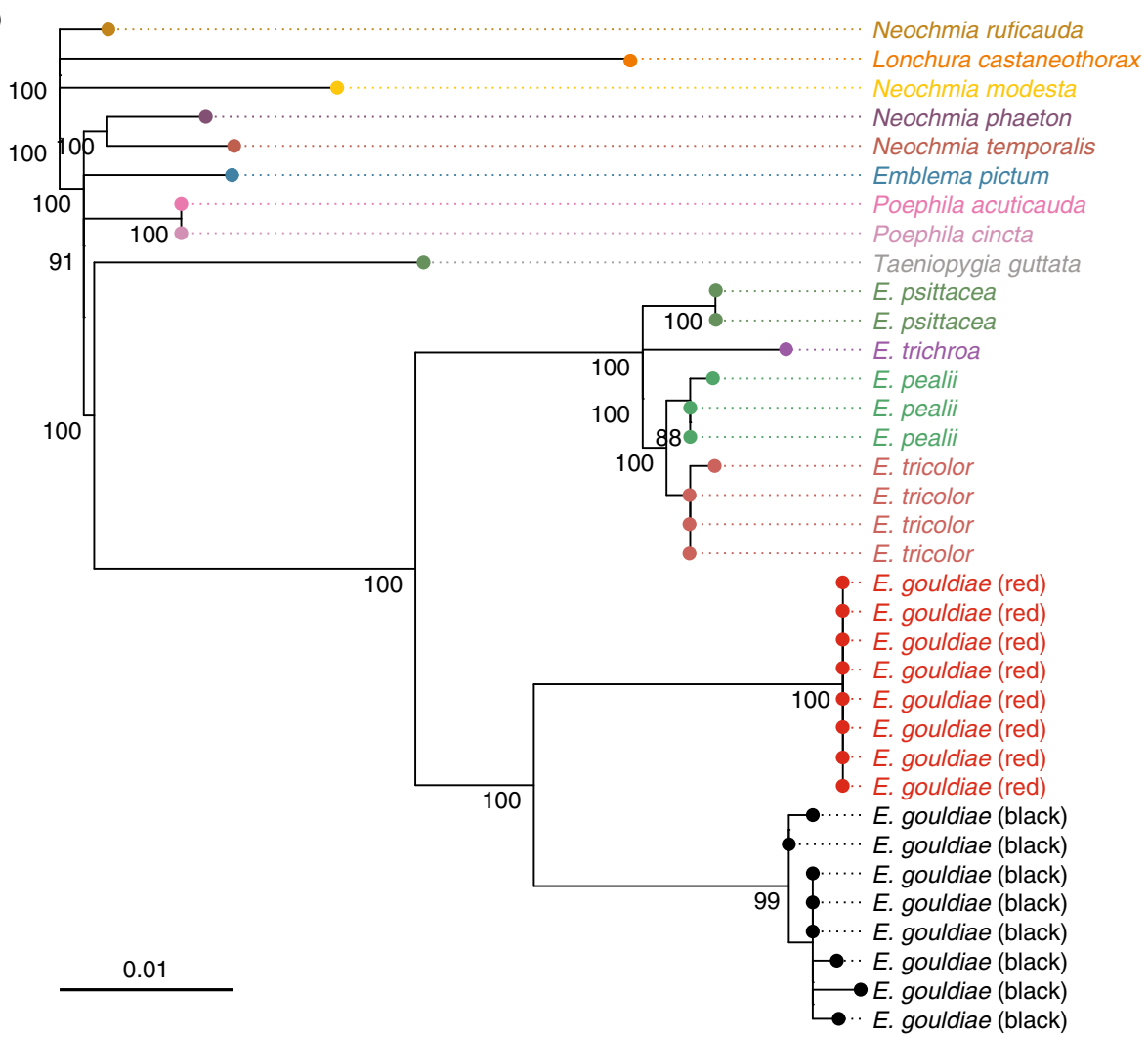

Fig. 4 Gene genealogy at the Red locus. a Illustrations of species in the genus Erythrura. From left, E. psittacea, E. trichroa, E. pealii, E. tricolor, and black and red morph of E. gouldiae (illustrations by Megan Bishop). b Maximum-likelihood tree $(\mathrm{GTR}+\Gamma+\mathrm{I})$ for the species in the family Estrildidae using 774 -bp alignment of M1 (Fig. 1). Node support values (\%) were generated from 1000 bootstrap replicates and values $>80 \%$ are shown next to the branches

insertion of the retrotransposon, which occurred some time after the divergence of Gouldian finch and zebra finch (Supplementary Fig. 19).

In a relative rate test, the red haplotype showed disproportionately longer branch lengths than the black haplotype across the Red locus (Supplementary Figs. 18 and 20), suggesting that the two haplotypes have evolved with unequal evolutionary rates. Although the driver of this asymmetry remains unclear, one possible explanation is that the red haplotype may have undergone more rounds of adaptive substitution, which would also explain the lower diversity of the red haplotype over a long stretch (Supplementary Figs. 9b and 10f).

The origin of divergence and the mode of balancing selection. There are several hypotheses for the origin of haplotypic divergence at the Red locus, including former allopatry followed by secondary contact ${ }^{43}$ or the lateral transfer of ancestral variation ${ }^{44}$. For example, in northern Australia, the historical Ord Arid Intrusion event resulted in the splitting of many co-distributed taxa into three distinct biogeographical populations, and some became (and remain) distinct subspecies ${ }^{45}$.
Regardless of the initial cause of divergence, our analyses suggest that balancing selection may be responsible for maintaining the divergent haplotypes at the Red locus. There are a few possible mechanisms of balancing selection in this species. First, red males may have an advantage under sexual selection, as females of all morphs of the Gouldian finch show a preference for red males ${ }^{35}$. Moreover, the dominant status of red males in the social structure ${ }^{46}$ may be beneficial when competing for limited resources, such as nest sites, albeit at the expense of reduced paternal care ${ }^{47}$. Since the red allele is genetically dominant, its allele frequency would be expected to quickly rise to a high level under such a selective advantage. However, the red phenotype is present at low frequency in natural populations ${ }^{12}$. Therefore, opposing selection has probably prevented the spread of the red allele in the population. Red males suffer from high stress levels and have significantly poorer reproductive outcomes than black birds when aggressive red males are in high frequency, and are consequently in a more competitive and stressful environment ${ }^{48-50}$. Thus, frequency-dependent selection may have acted to maintain a low equilibrium frequency of the red individuals. Alternatively, this may be an example of sexual antagonism $^{51}$, in which the fitness effects of different colouration 
are not the same in both sexes, and selection against red females prevents the red haplotype from increasing to a high frequency, despite being favoured in males. Red females fully express red head colour in captive populations (as a result of strong artificial selection by aviculturists). However, those in the wild population are less brightly coloured generally; the proportion of red feathers on their heads is typically lower, and they are often indistinguishable from black females ${ }^{9}$, suggesting selection against redness in females in the wild.

In this study, we have shown that a single locus upstream of FST is associated with colouration. This locus is intergenic, which does not support a role for a supergene in the classical sense in differentiating the red and black colour morphs of Gouldian finches. But it provides an example of a putative regulatory element with downstream effects on multiple phenotypes being maintained in the long-term by balancing selection. This has resulted in the maintenance of markedly divergent morphs, despite gene flow within the species, and in the absence of genomic rearrangements.

During the revision of the current manuscript we were alerted to a study on face colour polymorphism in domesticated Gouldian finches by Toomey et al. ${ }^{52}$. The research presented here confirms, complements, and extends that detailed study in several ways. First, both independent approaches highlight the same genomic region upstream of the follistatin gene, providing strong evidence of its functional role in generating the colour polymorphism. Second, unlike Toomey et al. ${ }^{52}$, our analysis incorporates data from wild birds, making clear the relevance of this region to non-domesticated populations. Finally, our simulations and evolutionary reconstructions add key historical context to how and why this polymorphism has evolved and is maintained in the wild.

The lack of wider genomic divergence between morphs suggests that the Red locus may not, alone, be sufficient to induce the incompatibilities observed in captive populations. This, in turn, suggests that the extended LD in captive populations (e.g. an $\sim 8 \mathrm{Mb}$ region showing zero recombination around the Red locus ${ }^{21}$ ) might encompass other linked loci underlying these incompatibilities. Therefore, further studies of the region around the Red locus will provide an important opportunity to address novel questions about the evolution of colour polymorphism. In particular, understanding the functional role of Red, a pleiotropic switch gene associated with both melanin and carotenoid colouration, may shed light on the mechanistic basis of sexual selection and genetic incompatibilities between colour morphs, which are both key evolutionary factors in speciation ${ }^{53}$.

\footnotetext{
Methods

DNA samples. Blood samples were collected from 161 Gouldian finches (male: $n_{\text {black }}=62, n_{\text {red }}=27$; female: $\left.n_{\text {black }}=64, n_{\text {red }}=8\right)$ in 2008 from a single wild population in Western Australia (within a $50-\mathrm{km}$ radius of Wyndham in East Kimberley). Birds were either caught in mist nets at waterholes or at their nests The population size was estimated to be around 300 adults. Genomic DNA was extracted using the ammonium acetate technique. The sex of each sample was determined using two sex-linked markers, $Z-002 D^{54}$ and $Z 37 B^{55}$ that amplify alleles on both the $\mathrm{Z}$ and $\mathrm{W}$ chromosomes. No power analysis was performed prior to the study. All 161 samples were used for the association test and a population structure analysis. A subset of samples was randomly selected and used for RADSeq and subsequent Sanger and MiSeq sequencing and genotyping (see below). Throughout the study, traits were measured blind to treatment and to values of other traits.

For whole-genome sequencing (WGS), blood samples were collected from 24 Gouldian finches (males: $n_{\text {black }}=4, n_{\text {red }}=4, n_{\text {yellow }}=3$; females: $n_{\text {black }}=3, n_{\text {red }}=$ $5, n_{\text {yellow }}=5$ ) that were sampled from a single captive population of domesticated birds in Ithaca, NY, in 2016. Yellow birds have the same allele at the Red locus as the red morph but develop as yellow under the control of an unknown epistatic autosomal locus ${ }^{11}$. Genomic DNA was extracted from these blood samples using DNeasy kits (tissue protocol; Qiagen, Hilden, Germany).
}

RADSeq. We genotyped 32 birds from the wild population described above using RADSeq $^{20}$ in two separate batches. In the first batch, DNA fragment libraries for 20 individuals $\left(n_{\text {black_male }}=10, n_{\text {red_female }}=8\right.$ and $\left.n_{\text {red_male }}=2\right)$ were constructed following restriction digestion with Sbfl, and the pooled libraries were sequenced using two lanes of Illumina Genome Analyser IIx. This sampling scheme was to identify the Red locus efficiently with limited sample size in GWAS (see below). In the second batch, a pooled library, including 12 additional black males from the same population, was constructed using the same protocol but sequenced on an Illumina HiSeq2000 instrument.

The quality of the short sequence reads $(2 \times 100 \mathrm{bp})$ was checked using FASTQC (http://www.bioinformatics.babraham.ac.uk/projects/fastqc/). We cleaned and deduplicated the sequence reads using the process_radtags and clone_filter utility programmes from STACKS ${ }^{56}$. We then mapped the sequence reads against the zebra finch (Taeniopygia guttata) reference genome assembly (taeGut3.2.4) using SeqMan NGen 4.0 (DNASTAR, Madison, Wisconsin, USA), allowing up to $15 \%$ divergence between sequence reads and the reference sequence. Single-nucleotide variants were called using SAMTOOLS v1.2 $2^{57}$ and filtered based on the minimum depth for each site per individual $(>2)$, minimum SNP quality (>250), maximum of missing genotypes across all individuals $(<30 \%)$ and minor allele frequency filter $(>0.05)$ using custom R scripts and VCFtools v0.1.12 $2^{58}$ Although the HiSeq2000 sequencing runs had more coverage than the GAIIx runs, by applying filtering based on missing genotypes we could effectively remove any sites that were found only in the HiSeq2000 run. Mean depth per locus in an individual was different among libraries (GAIIx: $\mathrm{L} 1=3.2, \mathrm{~L} 2=7.8$; HiSeq2000: $\mathrm{L} 3=13.2$ ). However, because we mixed equal numbers of black and red birds in $\mathrm{L} 1$ and L2, any bias due to the coverage in the inference of structure between morphs should be limited. Females were encoded to have only one allele for the genotypes of Z-linked loci using the $v c f$-fix-ploidy function of VCFtools. In an $F_{\mathrm{ST}}$ outlier analysis (Supplementary Fig. 1 ), we included 32 individuals $\left(n_{\text {black_male }}=22\right.$, $n_{\text {red_female }}=8$ and $n_{\text {red_male }}=2$ ) whose genotypes at the Red locus were either homozygous or hemizygous, except for the two heterozygous red males.

GWAS. SNPs linked to the causative site at the Red locus are expected to show a recessive homozygous genotype in black males $(a / a)$, to be hemizygous $(A)$ in red females and to be dominant homozygotes $(A / A)$ or heterozygotes $(A / a)$ in red males (see Introduction). In a genome-wide association study (GWAS) in 32 RAD sequenced samples, we used Fisher's exact tests with one degree of freedom for a dominant model of penetrance in a $2 \times 2$ contingency table of phenotype (black or red) by genotype ( $a / a$ or not $a / a$ ) using PLINK v1.959 (Fig. 1a). We treated the hemizygous Z-chromosome genotypes of females as homozygotes. We corrected for genomic structure among samples using the --within option and the familywise estimate of the SNP's significance was determined and corrected for multiple testing using the --mperm option (1000 permutations).

De novo low-coverage whole-genome sequencing. Sequencing libraries for the 24 captive Gouldian finches were generated using the Illumina TruSEQ PCR-free Library Preparation Kit following the protocol for generating 350-bp libraries. These 24 individuals were sequenced on a single lane of an Illumina NextSeq500 instrument to produce 150 -bp paired-end reads. We used AdapterRemoval ${ }^{60}$ to collapse overlapping paired reads, trim sequences of "Ns" along the 5 ' and 3 ' ends of reads, and remove sequences shorter than $20 \mathrm{bp}$. This resulted in a mean of 33.7 million total reads per individual (range 20.8-49.3 million reads). We used Bowtie $2^{61}$ to map each of the individual reads to the zebra finch genome to a mean coverage of $\sim 3.6 \times$, using the "very sensitive local" set of alignment presets. For SNP discovery and variant calling, we used the UnifiedGenotyper in GATK ${ }^{62}$. We removed possible variants that had "quality by depth" (QD) of $<2$ and "mapping quality" (MQ) of $<30$ (the filtering conditions were QD $<2.0$, FisherStrand $>40.0$, MQ $<30.0$, HaplotypeScore $>12.0$, MappingQualityRankSum $<-12.5$, ReadPosRankSum $<-8.0)$. Variant confidence is a measure of sequencing depth at a given variant site; mapping quality refers to the root-mean-square of the mapping confidence (from Bowtie 2) of reads across all samples. We applied additional filters using VCFtools. First, we coded genotypes with a Phred-scaled quality lower than 8 as missing data. This is a permissive filter, necessary given our low-coverage data, which allows for genotypic calls with as few as three reads at a given site for an individual. We excluded loci with more than $30 \%$ missing data and used a minor allele frequency filter of $5 \%$. This resulted in 4,818,133 SNPs distributed across the genome.

To properly correct for the ploidy of genotypes identified on the $\mathrm{Z}$ chromosome, we used the $v c f$-fix-ploidy function of VCFtools to assign one allele at each site for Z-linked loci in females. To increase the chance of detecting the location of the Red locus in an $F_{\mathrm{ST}}$ outlier analysis (Supplementary Fig. 1), we only included data from 17 individuals whose genotypes were known to be homozygous or hemizygous for alternative alleles at the Red locus based on the pedigree (black: $n_{\text {black male }}=4, n_{\text {black female }}=3$; red: $n_{\text {red female }}=5$ and $n_{\text {yellow female }}=5$ ).

Test for the association with colour phenotype. Several segregating sites associated with head colour were identified in the sequences around a RADSeq locus we found to be associated with head colour (locus Ego165, Supplementary Table 4). To confirm the association with plumage colour, 161 birds were genotyped for 
alternative two-base pair variants (TG/CA) using an allele-specific PCR with the Multiplex PCR Kit (QIAGEN) and a set of primers (locus M1, see Supplementary Table 2). The conditions for the PCR were $95^{\circ} \mathrm{C}$ for $15 \mathrm{~min}, 30$ cycles of $94^{\circ} \mathrm{C}$ for $30 \mathrm{~s}, 64^{\circ} \mathrm{C}$ for $90 \mathrm{~s}$ and $72{ }^{\circ} \mathrm{C}$ for $1 \mathrm{~min}$, followed by $72{ }^{\circ} \mathrm{C}$ for $5 \mathrm{~min}$. The PCR products were separated on an ABI 3730 DNA Analyser (Applied Biosystems, Foster City, California, USA) and scored using GENEMAPPER v 3.7 (Applied Biosystems). The association of the genotypes with face colour was tested for each sex using Fisher's exact tests in R v3.2.5 (R Core Team, 2015, Fig. 1b). In addition to the locus $M 1$, a multiplex marker set was developed including two allele-specific markers to genotype loci at the edges of the region of elevated LD at the Red locus (M2 and M3, Supplementary Table 2, Supplementary Fig. 2). The same condition as above was used in the PCR, except for an annealing temperature of $60^{\circ} \mathrm{C}$. We examined the deviation of the $M 1$ genotypes from Hardy-Weinberg equilibrium (HWE) using Graffelman-Weir's exact test for sex-linked markers implemented in the HardyWeinberg package ${ }^{63}$ and obtained the $P$-value by permuting individual genotypes 10,000 times (Supplementary Table 3).

The extent of LD and sequencing of a candidate gene. To determine the extent of $\mathrm{LD}$ across the Red locus and the association of marker loci with the colour trait, the DNA sequences of 26 short fragments in the upstream region and the whole exon of an adjacent candidate gene (follistatin) were obtained (Supplementary Table 4). We sequenced an additional 15 loci on the $\mathrm{Z}$ chromosome that are not close to the Red locus as controls (Supplementary Table 4). Genomic DNA from 16 females $\left(n_{\text {black }}=8, n_{\text {red }}=8\right)$ was amplified and cleaned with ExoSAP-IT (USB, Cleveland, Ohio, USA). After Sanger sequencing with the BigDye Terminator v 3.1 cycle sequencing kit (Applied Biosystems), and precipitation using the ethanol/ EDTA method, the products were separated on an ABI 3730 DNA Analyser and the bases were called using CodonCode Aligner v2.0.6 (CodonCode Corporation). Association tests between phenotype and 282 SNPs from 26 fragments, and the estimation of pairwise LD between SNPs, were performed using Haploview v4.264 and visualized using LDheatmap ${ }^{65}$ (Fig. 1c). The coding sequences of follistatin in 16 females were aligned, along with the homologous assembled zebra finch sequence, to examine the differences between species and morphs (Supplementary Fig. 4).

Test for the presence of an inversion. If there is an inversion breakpoint between two neighbouring loci, then the inverted chromosome will not produce amplicons across the breakpoint in a PCR that produces an amplicon in the non-inverted chromosome. To test for an inversion across the Red locus, 19 contiguous, overlapping fragments were amplified in 12 females $\left(n_{\text {black }}=6, n_{\text {red }}=6\right)$ from the wild population by combining each forward primer with a reverse primer designed within the adjacent amplicon (Supplementary Table 6) and using the LongRange PCR kit (QIAGEN). When the primer sets originally designed from zebra finch sequences failed to produce long amplicons, we designed new primers using the Gouldian finch sequences obtained from previous sequencing efforts to ensure the failure was not due to mismatching sequences in the primer-binding sites (Supplementary Table 6). We confirmed that the long fragments amplified the correct sites by sequencing the boundaries of each long fragment using PCR primers and comparing with the zebra finch reference genome.

Long amplicons across the Red locus from the above PCR experiments and short amplicons for the reference loci (see below) were labelled by individual, pooled and sequenced using the Illumina MiSeq platform. All traces of adapter sequences added to amplicons during paired-end short-read (250-bp) sequencing were removed using a custom Perl script (TagCle v0.80, available upon request from K.-W.K.). De novo assemblies were performed for each individual using NGen. Supercontigs were produced by BLAST searching the contig sequences against the zebra finch reference sequence and comparing the positions of the contigs and the overlapping sequences. The assembled sequences were aligned using UGENE v1.12.366 and manually edited using BioEdit v7.1.1167. These data were used to measure DNA polymorphism across the Red locus and in coalescent simulations to test for selection (see below).

Transcriptome analysis. We tested for differential gene expression in the feather follicles between the Gouldian finch morphs. First, we sampled regenerating head feathers from birds held by breeders in Australia during the annual moulting period and stored them directly in RNAlater (Qiagen). The feathers were subsequently frozen solid with liquid nitrogen, homogenized using a pestle and mortar, and RNA was extracted using TRIzol (Thermo Fisher Scientific, Waltham, Massachusetts, USA) and RNeasy Mini kits (Qiagen). The quality of the RNA was measured on a Bioanalyzer (Agilent, Santa Clara, California, USA) and highquality samples with an RNA integrity value $>7$ were used in microarray analysis. We obtained the gene expression profiles of six birds $\left(n_{\text {black_male }}=3, n_{\text {red_male }}=2\right.$, $n_{\text {red_female }}=1$ ) using the Affymetrix zebra finch gene array ST1.0 that contains probe sets from 18,595 genes. Gene expression was normalized using a robust multi-array average algorithm implemented in the Affymetrix Expression Console v1.3. The expression differences were tested using one-way ANOVA implemented in Transcriptome Analysis Console v2.0 (Affymetrix). Because of the small sample size, no gene passed the threshold required to correct for the false discovery rate. Therefore, we used uncorrected $P$-values $<0.05$ and $\log _{2}$-fold changes $\geq 1.3$ to select
304 potentially differentially expressed (DE) genes. Human orthologues of DE genes were obtained from UniProt (www.uniprot.org). A functionally grouped annotation network for the DE genes was constructed using the Cytoscape plugin ClueGO v2.3.5 ${ }^{68}$. Functionally related Gene Ontology (GO) terms for biological processes in human (23 February 2017) were grouped based on a kappa score $>0.4$ with network specificity of 3-5. The significance was examined using a two-sided hypergeometric test and the false discovery rate was corrected using the Benjamini-Hochberg method $^{69}$.

Evolutionarily conserved elements within the Red locus. We used PhastCons values $^{70}$ to examine the presence of the evolutionarily conserved elements within the Red locus that might represent regulatory regions potentially responsible for the expression of a candidate gene underlying colour polymorphism. The position of human and mouse transcripts for MOCS2 and FST, and the pre-calculated PhastCons values across the Red locus, were obtained from the UCSC Genome Browser Gateway (https://genome.ucsc.edu/cgi-bin/hgGateway). PhastCons values were based on multi-genome alignments among three vertebrates; medium ground finch (Geospiza fortis), human (Homo sapiens) and mouse (Mus musculus), and among five birds: medium ground finch, zebra finch, budgerigar (Melopsittacus undulatus), chicken (Gallus gallus) and turkey (Meleagris gallopavo). The records were visualized using $\mathrm{Gviz}^{71}$. The genomic position of the Red locus in the genome of the medium ground finch was determined using a BLAT search in the UCSC.

Genomic regions under consideration in the neutrality test. We obtained a 99,669-bp stretch of sequence using the Illumina MiSeq (see above) encompassing the Red locus $(46,503,706-46,601,744$ bp in zebra finch, Supplementary Table 6) in 12 female birds $\left(n_{\text {black }}=6, n_{\text {red }}=6\right)$ from the wild population. Because females are hemizygous at the Red locus, these sequences represent six each of the black $\left(\mathrm{Z}^{r}\right)$ and red $\left(\mathrm{Z}^{R}\right)$ alleles.

In an additional MiSeq run, we also randomly chose 24 loci (henceforth the 'reference' loci, Supplementary Table 8) across the Z chromosome, outside of the LD block of the Red locus, to measure the background level of diversity for comparison with that seen at the Red locus. These loci consisted of single introns, sampled in 11-12 female individuals, 10 of which (5 each of black and red) were also represented in our sample of 12 birds sequenced at the Red locus.

Estimates of DNA polymorphism and divergence. In order to compare diversity at the Red locus (combining the $\mathrm{Z}^{r}$ and $\mathrm{Z}^{R}$ alleles) with that at the reference loci using the MiSeq data, we calculated $\pi$ and Tajima's $D$ for the whole sample at each locus using a custom $\mathrm{R}$ script. We then calculated the same statistics within each allelic class (we define two allelic classes: one each for birds having the $\mathrm{Z}^{r}$ or the $\mathrm{Z}^{R}$ allele at the Red locus, respectively) at the reference loci and the Red locus, in order to compare polymorphism for the combined sample with that within each allelic class. In addition, we calculated $F_{\mathrm{ST}}$ and $D_{\mathrm{XY}}$ between the two allelic classes at the reference and the Red loci using custom R and Python scripts. We used Weir and

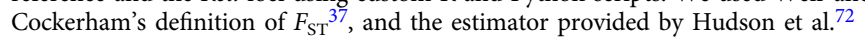
to calculate site-by-site $F_{\mathrm{ST}}$ values (Supplementary Fig. 1). To obtain a composite estimate of $F_{\mathrm{ST}}$ from multiple SNPs for each window, we used equation (6) of Jackson et al. ${ }^{73}$. In the estimation of $F_{\mathrm{ST}}, D_{\mathrm{XY}}$ and $\pi$ across the candidate region using sliding-window analyses (window size of $1000 \mathrm{bp}$ with a step of $500 \mathrm{bp}$; Figs. 2 and 3), we retained sites with missing data to maximise the number of SNPs in each window. However, because Tajima's $D$ cannot be calculated when data are missing, we removed individuals with missing data on a per window basis for this statistic.

We define statistics that refer to subsets of the data using subscripts: for example, $\pi_{\text {red }}$ refers to the nucleotide diversity among the alleles of red birds, and $\pi_{\text {total }}$ refers to nucleotide diversity among the alleles of both black and red birds combined. When conducting point estimates of summary statistics per locus we removed sites with missing data in any individual. To test for the statistical significance of the point estimate of Tajima's $D$, we used a coalescent simulation under the standard neutral model implemented in DnaSP v $5^{34}$.

To examine the genetic structure with regards to colour polymorphism, we constructed a coancestry matrix for samples for which we had RADSeq data that summarises the nearest-neighbour haplotype relationship and provides a more sensitive inference on population structure than the STRUCTURE and PCA approaches ${ }^{38,44}$ (Supplementary Fig. 6). We selected autosomal RADSeq loci with $\leq 5$ SNPs (total 18,719 RADSeq loci) and individuals with $\leq 20 \%$ missing data. We used fineRADstructure ${ }^{38}$ to obtain coancestry coefficients between individuals and data missingness for each individual. Two individuals had significantly high levels of missing data and were excluded from calculation of the coancestry matrix (Supplementary Fig. 6)

To examine the differentiation between morphs in all wild-population individuals, we used nine autosomal microsatellite markers from a set of previously-tested cross-species markers ${ }^{55}$ (Supplementary Table 10) and genotyped 161 birds $\left(n_{\text {black }}=126\right.$ and $n_{\text {red }}=35$ ) using the ABI3730 DNA Analyser. Deviation of microsatellite genotypes from HWE was examined using CERVUS v3.0.374. A model-based clustering method implemented in STRUCTURE v2.3.4. ${ }^{39}$ was used to infer the number of subpopulations $(K)$ and to assign individuals to populations 
(length of burnin period $=500,000$; number of MCMC repeats after burnin $=$ 750,000; 20 iterations for each run, Supplementary Fig. 7).

Considering nonrandom sampling in the test for selection. It is important to take into account nonrandom sampling with respect to the Red locus in simulations to generate polymorphism data under the null hypothesis of neutrality because, even under neutrality, it is possible to obtain high $F_{\mathrm{ST}}$ values, as well as elevated $\pi$ at a focal site, if the sampling scheme is conditioned on that site, as was the case here. In order to replicate our observed sampling scheme for the purpose of statistical testing, we used the allele frequencies from the genotypes of 161 wild birds at the Red locus for this purpose (Supplementary Table 3). As we do not know which allele $\left(Z^{r}\right.$ or $Z^{R}$ ) is ancestral, it was necessary to consider both scenarios in turn; i.e. $Z^{r}$ is derived (derived allele frequency, $\mathrm{DAF}=0.856$ ); or $Z^{R}$ is derived $(\mathrm{DAF}=0.144)$ (see Supplementary Methods for details about allele frequency estimation). This is, in part, because we cannot be sure which site(s) within the Red locus is causal, and so cannot polarise that site using an outgroup sequence. In addition, we lack outgroup sequence data for a portion of the Red locus that coincides with a putative TE insertion in the Gouldian finch lineage, but which does not exist at the same location in the zebra finch genome. As such, for the majority of the simulations and analyses below, we arbitrarily set the focal site at the centre of the Red locus. However, our results are robust to different choices of this parameter (see Supplementary Methods).

Testing for selection. We asked if the patterns of genetic variation we observed within the 100-kbp region are compatible with neutral evolution, given the fact that variants underlying the trait are in this region, and that our sampling is nonrandom. We employed two widely-used methods to test for evidence of natural selection. First, we used coalescent simulations to generate polymorphism data under neutrality at a locus that is equivalent to the $\sim 100$-kbp sequenced region in Gouldian finches in terms of its allele frequencies in nature (see above), the sampling scheme (6 each of red and black birds) and relevant population genetic parameters (the population mutation rate $\theta=0.0144$ estimated from 24 reference loci, and the population recombination rate $\rho=0.004$ estimated from the $\sim 100$-kbp sequenced region). Note that the simulations based on the 100-kbp region are robust to the estimate of the recombination rate because significant differentiation was also apparent between the red and black alleles in a simulation using $\rho=$ 0.0002 , which is $20 \times$ lower than the estimate from the 100 -kbp sequenced region $(\rho=0.004)$ (Supplementary Figs. 11 and 12). The lower value of $\rho$ approximates that inferred by LDhat ${ }^{75}$, both within the red and within the black allelic classes in the $100-\mathrm{kbp}$ region. It should be noted that the strong LD between the alleles prevented us from using LDhat to assay recombination in the combined sample. The conclusions from the simulations were also robust to limiting the range of sequence simulated to the 72-kbp Red locus (Supplementary Figs. 13 and 14). The estimate of $\rho$ from this region was unchanged from the value for the entire sequenced region. By assessing whether the observed polymorphism in the sequenced region is compatible with these simulations, we can accept or reject the null hypothesis of neutrality. We used the programmes SelSim ${ }^{76}$ and $\mathrm{mbs}^{77}$ to simulate polymorphism linked to a focal site at which a mutation has arisen and spread by genetic drift to the frequencies we observed at the Red locus $(0.144$ or 0.856). These programmes allowed us to replicate the allele frequencies, sampling scheme and population genetic parameters (see above) observed at the Red locus and obtain expectations under neutrality. In addition, mbs can model demographic changes. We also used a modified version of the HKA test ${ }^{41}$ to test for selection. See Supplementary Methods for more detailed methods.

Gene genealogy and relative rate test. To infer the evolutionary history of the haplotypes at the Red locus we constructed gene genealogies and compared the level of evolutionary divergence between the black and red haplotypes with those of orthologous sequences in other species. First, blood samples of 14 species in the family Estrildidae including red-faced (E. pealii, E. psittacae) or blue-faced (E. tricolor and E. trichroa) Erythrura species were obtained from an aviculturist. Blood samples were collected and stored using Whatman FTA cards (GE Healthcare, Little Chalfont, UK) and DNA was extracted following the manufacturer's instructions. Sequences of the fragments amplifiable using the M1-M3 primers (Supplementary Table 2) were obtained using Sanger sequencing. For zebra finch, the sequences from the reference genome assembly were used.

We tested alternative nucleotide substitution models using the $\mathrm{R}$ package, phangorn v2.3.178, which suggested GTR $+\Gamma+\mathrm{I}$ to be the best model for the sequence of the Red locus. Maximum-likelihood trees were then constructed using this model and tested with 1000 bootstraps using phangorn and visualised using ggtree v1.4.20 $0^{79}$. To examine if the molecular clock hypothesis applied to the Red locus, we used Tajima's nonparametric relative rate test implemented in pegas $\mathrm{v} 0.10^{80}$.

Compliance with ethical standards. Blood sampling of birds was conducted under the approval of the Animal Ethics Committees at the University of New South Wales and Macquarie University (ARAs 2007/037 \& 2007/038).

Reporting summary. Further information on research design is available in the Nature Research Reporting Summary linked to this article.

\section{Data availability}

The data that support the findings of this study are available from the corresponding authors on request. PLINK files for RADSeq analysis and sequence alignments of de novo sequencing for the Red locus underlying Figs. 1a and 4b, Supplementary Fig. 1, 2, 6 and 8-19 and Supplementary Tables 9 and 11-13 are available from the Dryad Digital Repository [https://doi.org/10.5061/dryad.9nk3757]. Raw Illumina reads are available at NCBI SRA (SAMN10751906-SAMN10751929) under BioProject PRJNA515277.

Microarray data are available on the Gene Expression Omnibus (GEO) under accession number GSE125295. A reporting summary for this article is available as a Supplementary Information file.

\section{Code availability}

Custom scripts are available from the corresponding authors on request.

Received: 9 April 2018 Accepted: 22 March 2019

Published online: 23 April 2019

\section{References}

1. Galeotti, P., Rubolini, D., Dunn, P. O. \& Fasola, M. Colour polymorphism in birds: causes and functions. J. Evol. Biol. 16, 635-646 (2003).

2. Hugall, A. F. \& Stuart-Fox, D. Accelerated speciation in colour-polymorphic birds. Nature 485, 631-634 (2012).

3. Roulin, A. The evolution, maintenance and adaptive function of genetic colour polymorphism in birds. Biol. Rev. 79, 815-848 (2004).

4. Gray, S. M. \& McKinnon, J. S. Linking color polymorphism maintenance and speciation. Trends Ecol. Evol. 22, 71-79 (2007).

5. Mundy, N. I. et al. Red carotenoid coloration in the zebra finch is controlled by a cytochrome P450 gene cluster. Curr. Biol. 26, 1435-1440 (2016).

6. Toomey, M. B. et al. High-density lipoprotein receptor SCARB1 is required for carotenoid coloration in birds. Proc. Natl Acad. Sci. USA 114, 5219-5224 (2017).

7. Cooke, T. F. et al. Genetic mapping and biochemical basis of yellow feather pigmentation in budgerigars. Cell 171, 427-439. e421 (2017).

8. Lopes, R. J. et al. Genetic basis for red coloration in birds. Curr. Biol. 26, 1427-1434 (2016).

9. Southern, H. N. Polymorphism in Poephila gouldiae Gould. J. Genet. 47, 51-57 (1945).

10. Brush, A. H. \& Seifried, H. Pigmentation and feather structure in genetic variants of the Gouldian finch, Poephila gouldiae. Auk 85, 416-430 (1968).

11. Cooke, F. \& Buckley, P. A. Avian Genetics: A Population and Ecological Approach. (Academic Press, Cambridge, 1987).

12. Gilby, A. J., Pryke, S. R. \& Griffith, S. C. The historical frequency of headcolour morphs in the Gouldian finch (Erythrura gouldiae). Emu 109, 222-229 (2009).

13. Bolton, P. E. et al. The colour of paternity: extra-pair paternity in the wild Gouldian finch does not appear to be driven by genetic incompatibility between morphs. J. Evol. Biol. 30, 174-190 (2017).

14. Schwander, T., Libbrecht, R. \& Keller, L. Supergenes and complex phenotypes. Curr. Biol. 24, R288-R294 (2014).

15. Lamichhaney, S. et al. Structural genomic changes underlie alternative reproductive strategies in the ruff (Philomachus pugnax). Nat. Genet. 48 $84-88$ (2016)

16. Küpper, C. et al. A supergene determines highly divergent male reproductive morphs in the ruff. Nat. Genet. 48, 79-83 (2016).

17. Tuttle, E. M. et al. Divergence and functional degradation of a sex chromosome-like supergene. Curr. Biol. 26, 344-350 (2016)

18. Kim, K.-W. et al. A sex-linked supergene controls sperm morphology and swimming speed in a songbird. Nat. Ecol. Evol. 1, 1168-1176 (2017).

19. Ducrest, A.-L., Keller, L. \& Roulin, A. Pleiotropy in the melanocortin system, coloration and behavioural syndromes. Trends Ecol. Evol. 23, 502-510 (2008).

20. Baird, N. A. et al. Rapid SNP discovery and genetic mapping using sequenced RAD markers. PLoS ONE 3, e3376 (2008).

21. Kim, K.-W., Griffith, S. C. \& Burke, T. Linkage mapping of a polymorphic plumage locus associated with intermorph incompatibility in the Gouldian finch (Erythrura gouldiae). Heredity 116, 409-416 (2016).

22. Stapley, J., Feulner, P. G. D., Johnston, S. E., Santure, A. W. \& Smadja, C. M. Variation in recombination frequency and distribution across eukaryotes: patterns and processes. Philos. Trans. R. Soc. Lond., B, Biol. Sci. 372, 20160455 (2017).

23. Ohyama, A., Saito, F., Ohuchi, H. \& Noji, S. Differential expression of two BMP antagonists, gremlin and Follistatin, during development of the chick feather bud. Mech. Dev. 100, 331-333 (2001).

24. McDowall, M., Edwards, N. M., Jahoda, C. A. B. \& Hynd, P. I. The role of activins and follistatins in skin and hair follicle development and function. Cytokine Growth F. R. 19, 415-426 (2008). 
25. Sharov, A. A. et al. Bone morphogenetic protein (BMP) signaling controls hair pigmentation by means of cross-talk with the melanocortin receptor-1 pathway. Proc. Natl Acad. Sci. USA 102, 93-98 (2005).

26. Moustakas, A. TGF- $\beta$ targets $P A X 3$ to control melanocyte differentiation. Dev. Cell 15, 797-799 (2008).

27. Yu, M., Wu, P., Widelitz, R. B. \& Chuong, C.-M. The morphogenesis of feathers. Nature 420, 308-312 (2002).

28. Belecky-Adams, T. L., Scheurer, D. \& Adler, R. Activin family members in the developing chick retina: expression patterns, protein distribution, and in vitro effects. Dev. Biol. 210, 107-123 (1999).

29. Moreira, E. F. \& Adler, R. Effects of follistatin overexpression on cell differentiation in the chick embryo retina. Dev. Biol. 298, 272-284 (2006).

30. Knight, P. G. \& Glister, C. Potential local regulatory functions of inhibins, activins and follistatin in the ovary. Reproduction 121, 503-512 (2001).

31. Toews, D. P. L. et al. Plumage genes and little else distinguish the genomes of hybridizing warblers. Curr. Biol. 26, 2313-2318 (2016).

32. Nei, M. Molecular Evolutionary Genetics. (Columbia Univ. Press, New York, 1987).

33. Tajima, F. Statistical method for testing the neutral mutation hypothesis by DNA polymorphism. Genetics 123, 585-595 (1989).

34. Librado, P. \& Rozas, J. DnaSP v5: a software for comprehensive analysis of DNA polymorphism data. Bioinformatics 25, 1451-1452 (2009).

35. Pryke, S. R. \& Griffith, S. C. The relative role of male vs. female mate choice in maintaining assortative pairing among discrete colour morphs. J. Evol. Biol. 20, 1512-1521 (2007).

36. Pryke, S. R. \& Griffith, S. C. Postzygotic genetic incompatibility between sympatric color morphs. Evolution 63, 793-798 (2009).

37. Weir, B. S. \& Cockerham, C. C. Estimating F-statistics for the analysis of population structure. Evolution 38, 1358-1370 (1984).

38. Malinsky, M., Trucchi, E., Lawson, D. J. \& Falush, D. RADpainter and fineRADstructure: population inference from RADseq data. Mol. Biol. Evol. 35, 1284-1290 (2018).

39. Pritchard, J. K., Stephens, M. \& Donnelly, P. Inference of population structure using multilocus genotype data. Genetics 155, 945-959 (2000).

40. Charlesworth, D. Balancing selection and its effects on sequences in nearby genome regions. PLoS Genet. 2, e64 (2006).

41. Hudson, R. R., Kreitman, M. \& Aguadé, M. A test of neutral molecular evolution based on nucleotide data. Genetics 116, 153-159 (1987).

42. Van't Hof, A. E. et al. The industrial melanism mutation in British peppered moths is a transposable element. Nature 534, 102 (2016).

43. Garrigan, D., Mobasher, Z., Kingan, S. B., Wilder, J. A. \& Hammer, M. F. Deep haplotype divergence and long-range linkage disequilibrium at Xp21.1 provide evidence that humans descend from a structured ancestral population. Genetics 170, 1849-1856 (2005).

44. Stryjewski, K. F. \& Sorenson, M. D. Mosaic genome evolution in a recent and rapid avian radiation. Nat. Ecol. Evol. 1, 1912-1922 (2017).

45. Bowman, D. M. J. S. et al. Biogeography of the Australian monsoon tropics. J. Biogeogr. 37, 201-216 (2010).

46. Pryke, S. R. \& Griffith, S. C. Red dominates black: agonistic signalling among head morphs in the colour polymorphic Gouldian finch. Proc. R. Soc. B 273, 949-957 (2006).

47. Brazill-Boast, J., Griffith, S. \& Pryke, S. Morph-dependent resource acquisition and fitness in a polymorphic bird. Evol. Ecol. 27, 1189-1198 (2013).

48. Pryke, S. R., Astheimer, L. B., Buttemer, W. A. \& Griffith, S. C. Frequencydependent physiological trade-offs between competing colour morphs. Biol. Lett. 3, 494-497 (2007).

49. Pryke, S. R. \& Griffith, S. C. Socially mediated trade-offs between aggression and parental effort in competing color morphs. Am. Nat. 174 455-464 (2009).

50. Pryke, S. R., Astheimer, L. B., Griffith, S. C. \& Buttemer, W. A. Covariation in life-history traits: differential effects of diet on condition, hormones, behavior, and reproduction in genetic finch morphs. Am. Nat. 179, 375-390 (2012).

51. Arnqvist, G. \& Rowe, L. Sexual Conflict. (Princeton University Press, New Jersey, 2005).

52. Toomey, M. B. et al. A non-coding region near Follistatin controls head colour polymorphism in the Gouldian finch. Proc. R. Soc. B 285, 20181788 (2018).

53. Price, T. D. Speciation in Birds. (Roberts and Company, 2008).

54. Dawson, D. A. Genomic Analysis of Passerine Birds Using Conserved Microsatellite Loci PhD Thesis. (University of Sheffield, UK, 2007).

55. Kim, K.-W. et al. Four-way development of microsatellite markers for the Gouldian finch (Erythrura gouldiae). Conserv. Genet. Resour. 7, 899-907 (2015).

56. Catchen, J. M., Amores, A., Hohenlohe, P., Cresko, W. \& Postlethwait, J. H. Stacks: building and genotyping loci de novo from short-read sequences. G3Genes Genom. Genet. 1, 171-182 (2011).

57. Li, H. A statistical framework for SNP calling, mutation discovery, association mapping and population genetical parameter estimation from sequencing data. Bioinformatics 27, 2987-2993 (2011).
58. Danecek, P. et al. The variant call format and VCFtools. Bioinformatics 27, 2156-2158 (2011)

59. Purcell, S. et al. PLINK: a tool set for whole-genome association and population-based linkage analyses. Am. J. Hum. Genet. 81, 559-575 (2007).

60. Lindgreen, S. AdapterRemoval: easy cleaning of next-generation sequencing reads. BMC Res. Notes 5, 337 (2012).

61. Langmead, B. \& Salzberg, S. L. Fast gapped-read alignment with Bowtie 2. Nat Methods 9, 357 (2012).

62. McKenna, A. et al. The Genome Analysis Toolkit: a MapReduce framework for analyzing next-generation DNA sequencing data. Genome Res. 20, 1297-1303 (2010)

63. Graffelman, J. Exploring diallelic genetic markers: the HardyWeinberg package. J. Stat. Softw. 64, 1-23 (2015).

64. Barrett, J. C., Fry, B., Maller, J. \& Daly, M. J. Haploview: analysis and visualization of LD and haplotype maps. Bioinformatics 21, 263-265 (2005).

65. Shin, J.-H., Blay, S., McNeney, B. \& Graham, J. LDheatmap: an R function for graphical display of pairwise linkage disequilibria between single nucleotide polymorphisms. J. Stat. Softw. 16, 9 (2006).

66. Okonechnikov, K., Golosova, O. \& Fursov, M. Unipro UGENE: a unified bioinformatics toolkit. Bioinformatics 28, 1166-1167 (2012).

67. Hall, T. A. BioEdit: a user-friendly biological sequence alignment editor and analysis program for Windows 95/98/NT. Nucl. Acid. S. 41, 95-98 (1999).

68. Bindea, G. et al. ClueGO: a Cytoscape plug-in to decipher functionally grouped gene ontology and pathway annotation networks. Bioinformatics $\mathbf{2 5}$, 1091-1093 (2009).

69. Benjamini, Y. \& Hochberg, Y. Controlling the false discovery rate: a practical and powerful approach to multiple testing. J. R. Stat. Soc. Ser. B Stat. Methodol. 57, 289-300 (1995).

70. Siepel, A. et al. Evolutionarily conserved elements in vertebrate, insect, worm, and yeast genomes. Genome Res. 15, 1034-1050 (2005).

71. Hahne, F. \& Ivanek, R. in Statistical Genomics: Methods and Protocols Vol. 1418, 335-351 (Humana Press, 2016).

72. Hudson, R. R., Slatkin, M. \& Maddison, W. P. Estimation of levels of gene flow from DNA sequence data. Genetics 132, 583-589 (1992).

73. Jackson, B. C., Campos, J. L. \& Zeng, K. The effects of purifying selection on patterns of genetic differentiation between Drosophila melanogaster populations. Heredity 114, 163-174 (2015).

74. Kalinowski, S. T., Taper, M. L. \& Marshall, T. C. Revising how the computer program Cervus accommodates genotyping error increases success in paternity assignment. Mol. Ecol. 16, 1099-1106 (2007).

75. Auton, A. \& McVean, G. Recombination rate estimation in the presence of hotspots. Genome Res. 17, 1219-1227 (2007).

76. Spencer, C. C. A. \& Coop, G. SelSim: a program to simulate population genetic data with natural selection and recombination. Bioinformatics 20, 3673-3675 (2004).

77. Teshima, K. M. \& Innan, H. mbs: modifying Hudson's ms software to generate samples of DNA sequences with a biallelic site under selection. BMC Bioinforma. 10, 166 (2009).

78. Schliep, K. P. phangorn: phylogenetic analysis in R. Bioinformatics 27, 592-593 (2011)

79. Yu, G., Smith, D. K., Zhu, H., Guan, Y. \& Lam, T. T.-Y. ggtree: an r package for visualization and annotation of phylogenetic trees with their covariates and other associated data. Methods Ecol. Evol. 8, 28-36 (2017).

80. Paradis, E. pegas: an R package for population genetics with an integrated-modular approach. Bioinformatics 26, 419-420 (2010).

\section{Acknowledgements}

We thank Brian and Deborah Charlesworth for comments on an earlier version of the manuscript. Sarah Pryke and David Harris provided blood samples of the Gouldian finches and other species of Estrildidae. Peri Bolton assisted with the collection of cheek feathers. We thank the Natural Environment Research Council Biomolecular Analysis Facility (NBAF) at the University of Edinburgh for sequencing RAD libraries (NBAF674) and NBAF at the University of Liverpool for gene expression microarray scanning. This study was supported by a University of Sheffield scholarship to K.-W.K., a joint University of Sheffield/Natural Environmental Research Council (NE/H524881/1, NE/ K500914/1) Ph.D. studentship to B.C.J., a UK Department for Business, Innovation and Skills/Ministry of Education of China UK-China Scholarships for Excellence studentship to H.Z., an NSERC Banting postdoctoral fellowship to D.P.L.T., an Australian Research Council (DP130100418) grant to S.C.G. and T.B., and a Leverhulme Fellowship to T.B.

\section{Author contributions}

K.-W.K., B.C.J., S.C.G., K.Z. and T.B. conceived and designed the study. S.C.G. managed the work on Gouldian finches in the wild in Australia. K.-W.K., M.M.L. and A.D. constructed the RAD library. D.P.L.T, S.A.T., E.I.G and I.J.L. performed whole-genome sequencing. K.-W.K. and H.Z. performed molecular work. K.-W.K. and B.C.J. analysed the data. K.-W.K. and B.C.J. wrote the paper with contributions from all other authors 


\section{Additional information}

Supplementary Information accompanies this paper at https://doi.org/10.1038/s41467019-09806-6.

Competing interests: The authors declare no competing interests.

Reprints and permission information is available online at http://npg.nature.com/ reprintsandpermissions/

Journal peer review information: Nature Communications thanks the anonymous reviewers for their contribution to the peer review of this work. Peer reviewer reports are available.

Publisher's note: Springer Nature remains neutral with regard to jurisdictional claims in published maps and institutional affiliations. (c) (i) Open Access This article is licensed under a Creative Commons Attribution 4.0 International License, which permits use, sharing, adaptation, distribution and reproduction in any medium or format, as long as you give appropriate credit to the original author(s) and the source, provide a link to the Creative Commons license, and indicate if changes were made. The images or other third party material in this article are included in the article's Creative Commons license, unless indicated otherwise in a credit line to the material. If material is not included in the article's Creative Commons license and your intended use is not permitted by statutory regulation or exceeds the permitted use, you will need to obtain permission directly from the copyright holder. To view a copy of this license, visit http://creativecommons.org/ licenses/by/4.0/.

(C) The Author(s) 2019 